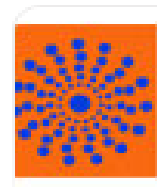

JOURNAL OF BEHAVIOR, hiEAth de SOCIAL issura

inis
Journal of Behavior, Health \& Social Issues ISSN: 2007-0780

jcpedro@unam.mx

Asociación Mexicana de Comportamiento y Salud, A. C.

México

Castellanos-Meza, Felix Alejandro; Torres-Ceja, Carlos de Jesús; Ortiz-Rueda, Gerardo Alfonso EL COMPORTAMIENTO HIPNÓTICO COMO UN EPISODIO SUSTITUTIVO REFERENCIAL Journal of Behavior, Health \& Social Issues, vol. 5, núm. 1, mayo-octubre, 2013, pp. 11-24 Asociación Mexicana de Comportamiento y Salud, A. C. Distrito Federal, México

Disponible en: http://www.redalyc.org/articulo.oa?id=282227878002

Cómo citar el artículo

- Número completo

- Más información del artículo

- Página de la revista en redalyc.org

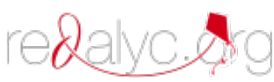

Sistema de Información Científica

Red de Revistas Científicas de América Latina, el Caribe, España y Portugal Proyecto académico sin fines de lucro, desarrollado bajo la iniciativa de acceso abierto 


\title{
EL COMPORTAMIENTO HIPNÓTICO COMO UN EPISODIO SUSTITUTIVO REFERENCIAL
}

\author{
HYPNOTIC BEHAVIOR AS A REFERENTIAL \\ SUBSTITUTION EPISODE
}

\section{Felix Alejandro Castellanos-Meza \\ Carlos de Jesús Torres-Ceja \\ Gerardo Alfonso Ortiz-Rueda}

Universidad de Guadalajara, Guadalajara, México

\begin{abstract}
Recibido: Junio 29, 2012
Revisado: Agosto 21, 2012

Aprobado: Enero 24, 2013

El primer autor agradece el apoyo recibido por parte del Consejo Nacional de Ciencia y Tecnología (Beca 206679) en la realización de esta investigación. El presente estudio fue conducido como parte de las actividades en el posgrado bajo la dirección del Dr. Carlos de Jesús Torres Ceja. También agradece las sugerencias del Dr. Gerardo Ortiz. Dirigir la correspondencia al primer autor, correo electrónico: felixacastellanosmayahoo.com.mx"
\end{abstract}

Resumen

El análisis del comportamiento hipnótico ha involucrado perspectivas en las que se ha privilegiado el rol de los factores biológicos o sociales, dejando a un lado los aspectos psicológicos o sometiéndolos a una lógica de causalidad lineal. Esto no ha favorecido una comprensión adecuada de los fenómenos hipnóticos y ha perpetuado algunas confusiones a lo largo de la historia. Desde una perspectiva interconductual (Ribes \& López, 1985) el presente documento analiza el problema de los eventos psicológicos denominados como "hipnosis" en el lenguaje ordinario. Se propone que la hipnosis puede conceptuarse como eventos de Afectación Paradójica Estable (o eventos APE), en los que se requiere el lenguaje para la ocurrencia de un desligamiento funcional de las propiedades situacionales presentes, por lo que el comportamiento hipnótico podría ubicarse en la categoría de Función Sustitutiva Referencial. Se ha tomado al fenómeno de la "anestesia/analgesia hipnótica" como situación caso, para exponer la articulación de las relaciones funcionales que participan en la facilitación del comportamiento hipnótico.

Palabras clave: Comportamiento hipnótico, hipnosis, función sustitutiva referencial, analgesia hipnótica, sugestión hipnótica. 
Abstract

The analysis of the hypnotic behavior has involved perspectives focused on the biological or social factors. The psychological aspects have been left aside and put under a logic of lineal causality. This has obstructed an adequate understanding about the hypnotic phenomena and has perpetuated some confusion. From an interbehavioral perspective (Ribes \& López, 1985) the present work analyses the problem of the psychological events called "hypnosis" in the ordinary language. It proposes that, hypnosis can be conceived as events of Paradoxical Stable Affectation (or PSA events). In these events, language promotes the occurrence of a functional unbinding from the present situational properties, that is why the hypnotic behavior could be classified in the Referential Substitutive Function. The phenomenon known as "hypnotic anesthesia/analgesia" has been chosen as case situation to show the articulation of the functional relations that participate in the facilitation of the hypnotic behavior.

Key words: Hypnotic behavior, Hypnosis, Referential Substitutive Function, Hypnotic analgesia, hypnotic suggestion.

Introducción

"Vaga por dentro y por fuera de los laboratorios y carnavales y clínicas y salones pueblerinos, como anomalía indeseada". (Jaynes, 1976, p. 379).

El tema de la hipnosis con frecuencia involucra actitudes desfavorables por parte de algunos académicos, que lo enfrentan de de los prejuicios formados respecto de la poca seriedad que se asume del área (Bernheim, 1887; Jaynes, 1976; Yapko, 1995). No obstante, es posible identificar que los eventos psicológicos agrupados bajo la nomenclatura "hipnosis" se vinculan con el seguimiento de instrucciones, la relación entre el decir y el hacer, y la conformación de los eventos privados (Cangas, 1998). Desde sus inicios, el tópico de la hipnosis ha involucrado la exploración acerca de cómo el lenguaje puede modificar las reacciones fisiológicas habituales como, por ejemplo, cuando un sujeto padece alguna enfermedad o afectación física (e.g. Bernheim, 1887; Elliotson, 1843; Kroger 1963; Pavlov, 1929; Platonov, 1959; Wundt, 1892). Algunos efectos similares pueden encontrarse en la utilización de técnicas de relajación y de retroalimentación biológica (Vázquez-Mata, Vital-Flores, Bravo-González, Jurado-Cárdenas \& Reynoso-Erazo, 2009). En la actualidad, es posible observar que el uso de la hipnosis se ha vinculado con la ejecución de respuestas efectivas, como una condición que facilita la precisión y un desempeño óptimo de acuerdo a tareas específicas (e.g. Morgan, 2002; Morgan \& Stegner, 2008; Pates, Maynar \& Westbury, 2001; Pates, Oliver \& Maynard, 2001)

El estudio del comportamiento hipnótico ha implicado diversas formas de abordajes a lo largo de más de doscientos años, cuestión que ha dado pie a múltiples confusiones teóricas, debido a las polarizaciones que se dan por las explicaciones basadas en uno u otro aspecto que interviene en el fenómeno (Dixon \& Laurence, 1992). Esto ha orientado el estudio del comportamiento hipnótico hacia los aspectos biológicos (Crawford \& Gruzelier, 1992; Pavlov, 1929; Platonov, 1959; Wundt, 1892), lo social (Sarbin \& Coe, 1972; Spanos \& Coe, 1992), los procesos o mecanismos cognoscitivos (Hilgard, 1973, 1992; Kirsch \& Lynn, 1998), la observación conductal, utilizando una lógica lineal causa-efecto (Barber, 1969; Skinner, 1957) o a las explicaciones eclécticas, donde se tornan irrelevantes las distinciones entre los tipos de funciones que poseen los diversos elementos (e.g. Erickson, Rossi \& Rossi, 1976; Gruzelier, 2000).

Las tendencias anteriores han perpetuado varios malentendidos, como por ejemplo, Ilamar "hipnosis" a distintos tipos de situaciones (Yapko, 1995) o tratar a "la hipnosis" como si fuera una sola cosa unitaria, en lugar de un conjunto de cosas de diferentes tipos (Hull, 1933), entre otros. Así, se ha señalado que el estudio del comportamiento hipnótico ha padecido de confusiones en al menos tres ámbitos diferentes: en la articulación de 
conceptos, en la lógica utilizada y la metodología empleada (Castellanos, 2009).

En cuanto a la articulación del concepto de "hipnosis", es posible apreciar que hay una mezcla de vocablos aparentemente técnicos con términos del lenguaje ordinario, como en el caso de "la sugestión", además de incluir fenómenos que aparentan formar parte de lo que se ha denominado como "hipnosis" (Castellanos, 2009). Esto Ileva a cometer errores categoriales - situar el uso de un término en una categoría diferente a la que pertenece (Ryle, 1949)-, así como a generar contradicciones y dificultades al tratar de entender cómo es que se integran las situaciones llamadas "hipnosis" (Dixon \& Laurence, 1992; González Ordi \& Miguel Tobal, 1994)

Sin importar la preferencia teórica por alguna categoría de factores (biológicos, verbales o sociales) que interviene en la formación de los eventos hipnóticos, las explicaciones en torno a la ocurrencia de los fenómenos hipnóticos tienen como base una lógica organocéntrica -o centrada en el organismo (Ribes, 1990a)- que implica considerar al individuo o los actos del individuo como eje central de interés, sin tener en cuenta la manera en que se articulan los sistemas contingenciales. Esto ha derivado en un análisis molecular de los fenómenos hipnóticos (concentrado en algún componente específico) sin considerar que los eventos psicológicos implican eventos molares - O sucesos continuos en espacio y tiempo, constituyendo campos de interacciones múltiples- (Ribes \& López, 1985).

Las confusiones conceptuales y lógicas han contribuido a la elaboración de instrumentos o escalas que funcionan bajo una lógica de causalidad lineal y pretenden medir conceptos que, de inicio, no responden a logros específicos, sino a sistemas de relaciones funcionales [véase como ejemplos, la Escala de Susceptibilidad Hipnótica de Stanford Tipo C (Weitzenhoffer \& Hilgard, 1962) o la Escala de Sugestionabilidad de Barber (Barber, 1969)].

Al considerar los elementos mencionados, se torna pertinente señalar que el problema de la hipnosis dentro del análisis conductual, ha padecido varias deficiencias. Entre éstas se encuentran: la estigmatización académica del tema, la confusión de situaciones que corresponden a distintas clases de sistemas contingenciales, la marginación de los aspectos conductuales que intervienen en los eventos psicológicos y la concentración de los estudios en aspectos orgánicos, mentalistas o sociales, y el uso de instrumentos que perpetúan una lógica de causalidad lineal. El objetivo del presente trabajo es mostrar que, desde una perspectiva interconductual (Ribes \& López, 1985), se puede articular un abordaje más adecuado hacia el problema del comportamiento hipnótico. Para esto se procederá, en primer lugar, a exponer los aspectos generales de Teoría de la Conducta (Ribes \& López, 1985), y en segundo, a hacer énfasis en la categoría denominada Función Sustitutiva Referencial. Después, se exhibirá cómo el comportamiento hipnótico puede ser considerado como un evento de Afectación Paradójica Estable, perteneciente al nivel de la Función Sustitutiva Referencial. Posteriormente se abordará el fenómeno hipnótico de la Anestesia/Analgesia como una situación caso de la Función Sustitutiva Referencial. Para finalizar, se presentarán las conclusiones del presente trabajo.

\section{Teoría de la conducta}

La visión interconductista (Ribes \& López, 1985) parte de una lógica de campo, donde el concepto de lo psicológico se traduce en un sistema de relaciones funcionales. Ribes $\left(1990^{\mathrm{a}}, 1994\right)$ señala las ventajas que posee esta manera de abordar los eventos psicológicos en comparación a otras propuestas psicológicas. Una de ellas es que se posibilita un análisis donde los elementos funcionales no se organizan de manera lineal causa-efecto, sino que articulan sistemas contingenciales donde varios factores de distinto tipo intervienen simultáneamente en la estructuración de un evento psicológico. Esto permite considerar la intervención de aspectos biológicos y sociales en la articulación de los eventos psicológicos, mientras se conserva la observación en los dominios propios de la psicología. Ribes y López (1985) han señalado que los aspectos biológicos son necesarios, mas no suficientes, para la formación de los eventos psicológicos y que los aspectos sociales, aunque participan en los eventos psicológicos, corresponden a una categoría diferente que no puede reducirse a lo psicológico o viceversa. De esta manera, aunque 
se pueden considerar elementos que pertenecen a distintas categorías, estos no se confunden con los factores psicológicos que articulan los eventos de hipnosis como sucede, por ejemplo, cuando se supone que un determinado funcionamiento cerebral produce los fenómenos hipnóticos (Wundt, 1892) o que los fenómenos hipnóticos son consecuencia de la adopción de un rol en términos sociales (Sarbin \& Coe, 1972).

La taxonomía de Ribes y López (1985) está constituida por cinco categorías que representan niveles de complejidad en las relaciones funcionales que integran sistemas de contingencias. Estos niveles se articulan mediante una lógica incluyente, donde las categorías funcionales más sencillas se encuentran implícitas en las más complejas. A grandes rasgos, los niveles propuestos en dicha taxonomía son: Función Contextual (fCx), donde el estímulo terminal media una relación funcional de tipo isomórfica que permite un ajuste conductual diferencial, mediante el desligamiento temporal de la respuesta. Función Suplementaria (fSu), que implica a una respuesta del organismo articulando una relación operativa, mientras permite un ajuste conductual basado en el desligamiento espacial y temporal de la respuesta. Función Selectora (fSe), constituida desde una relación entre dos estímulos que cambian y establecen permutaciones, sin importar las propiedades absolutas de los mismos, mientras un ajuste conductual basado en la precisión se hace posible. Función Sustitutiva Referencial (fSR), cuando una respuesta convencional - o lingüística - articula una relación entre dos contingencias - o situaciones- y se desvanecen las propiedades situacionales de los objetos presentes, porque hay un tránsito desde otra situación y la sustitución de contingencias resulta congruente. Función Sustitutiva No Referencial (fSNR), articulada por una respuesta convencional relacional que media una relación reflexiva, a partir del desligamiento absoluto de las propiedades físicas, permitiendo un ajuste basado en la coherencia de los eventos lingüísticos (Ribes \& López, 1985, Ribes, 2004, 2007).

Para los fines del presente trabajo, se obviarán los detalles de las Ilamadas funciones presustitutivas (véase las funciones Contextual, Suplementaria y Selectora), así como de la Función Sustitutiva No Referencial (fSNR). El texto se abocará a la descripción de la Función Sustitutiva Referencial (fSR) - para mayor detalle, consúltese Ribes y López (1985)-.

La Función Sustitutiva Referencial -fSREn la obra Teoría de la Conducta (Ribes \& López, 1985), se ha señalado que la articulación de la Función Sustitutiva Referencial (fSR) consta de las siguiente condiciones: a) un medio de contacto convencional (o lenguaje); b) contactos que requieren de dos momentos - situaciones- de respuesta (que pueden o no involucrar dos organismos, que puede implicar a las respuestas de dos individuos o dos respuestas de un mismo individuo en momentos diferentes); c) el desligamiento funcional de las propiedades situacionales espacio-tiempo de los eventos con los que se interactúa, mediante el uso del sistema reactivo convencional; $y, d$ ) la emergencia de contingencias de ocurrencia que se identifican al ajuste del sistema o función.

La articulación de la fSR implica una respuesta convencional, o lingüística en el sentido que denota Ribes (2006), que media una relación de transitividad entre los elementos que van de una situación a otra, así como el desligamiento de las propiedades situacionales que permite un ajuste conductual basado en la congruencia actuándose en una situación como si se estuviera en otra (Ribes \& López, 1985; Ribes, 2004, 2007). De acuerdo con Ribes (1992), al perderse la operatividad física en un sistema de contingencias "se rompen los límites de la situacionalidad, y el tiempo y el espacio operan como dimensiones múltiples y extrasituacionales (...) El aquí y el ahora sólo significan algo en términos del antes, el después y el allá" (p.82).

En este sentido, Torres, Ortiz, Rangel y González (2012) señalan que la articulación de la fSR implica un sistema de contingencias asociado a prácticas sociales, donde se presenta un contacto por traslación de ubicación de las formas de relación pertinentes: "Los sistemas de contingencia posibilitados a partir de la dimensión convencional suponen la estructuración de por lo menos dos situaciones. Una en que se pueden identificar eventos y acontecimientos delimitados espaciotemporalmente $(\mathrm{S} 1, \mathrm{Sn})$ pero cuyas relaciones de condicionalidad se encuentren determinadas por relaciones de contingencias identificadas 
como sociales (Sc)" (p.161). En el caso de la fSR, ambas situaciones, entendidas como sistemas de contingencias, están vigentes simultáneamente (Torres et al., 2012).

Al hablar sobre la fSR, es necesario considerar el aspecto de las Interacciones Extrasituacionales (e.g. Ortiz, Quintana \& Torres, 2009; Ribes, 1990b): actuar frente a los elementos y contingencias presentes, como si poseyeran las características o propiedades de otros elementos o contingencias. Implicaría, por tanto, reaccionar como sí una cosa fuera otra, reaccionar ante algo como si no estuviera, reaccionar ante la ausencia de algo como si estuviera, reaccionar como si lo inmediato fuera distinto, padecer el efecto de algo que no está, o las combinaciones que pudieran suceder ante el concilio de los observadores de un evento que les resulta ajeno (un evento privado que el sujeto experimental demuestra). Lo anterior sugiere que los eventos psicológicos agrupados bajo la nomenclatura de "comportamiento hipnótico" pueden articularse mediante los elementos expuestos bajo la lógica interconductista (Ribes \& López, 1985).

\section{El comportamiento hipnótico o eventos $A P E$ como un caso de fSR}

Antes de analizar el comportamiento hipnótico como un caso de la función sustitutiva referencial, se torna pertinente señalar que, a pesar de que se ha llegado a hablar de la hipnosis animal (i.e. en gallinas y perros, entre otras especies), es necesario establecer que se trata, básicamente, de una alegoría que hace referencia a la inmovilización de los animales mediante desorientación espacial, la exposición de situaciones de riesgo o el uso de estimulación táctil (Gilman \& Marcase, 1949). Cuando se dice que los animales son "hipnotizables", es en el sentido metafórico de antropomorfizar la capacidad fisiológica de otras especies para evocar una condición similar a la función neurológica del sueño: un aspecto que es necesario, mas no suficiente, para articular el concepto de hipnosis como se hace en el caso del hombre, es decir, una serie de afectaciones promovidas mediante el lenguaje (Ribes, 1990a).

Al tener presentes las cuatro condiciones necesarias para establecer la ocurrencia de un evento psicológico enmarcado dentro de la fSR (Ribes y
López, 1985), se puede observar el cumplimiento de estos en la articulación de un evento denominado "hipnosis":

1- Las interacciones se dan a través de un sistema reactivo convencional. Se puede observar que, desde los primeros estudios formales acerca del comportamiento hipnótico, se menciona que las alteraciones fisiológicas (exhibidas por los individuos en el estado hipnótico), son evocadas mediante las palabras que el operador utiliza (Bernheim, 1887; Wundt, 1892), al tiempo que se ha señalado que un dominio lingüístico adecuado para evocar fenómenos hipnóticos puede exhibirse en niños, a partir de los 5 años de edad (Kroger, 1963; Yapko, 1995). De hecho, Pavlov (1929) hizo mención del lenguaje como un factor necesario para evocar los fenómenos que caracterizan al comportamiento hipnótico en el humano, a diferencia de los animales. Bajo tal propuesta, Platonov (1959) demostró que era posible evocar alteraciones fisiológicas en funciones autónomas como la presión arterial y las contracciones musculares durante el trabajo de parto.

2- La interrelación entendida como contactos que requieren de dos momentos de respuesta (que pueden o no implicar 2 organismos). En el caso del comportamiento hipnótico, es necesario que las circunstancias promovidas por un operador logren mediar las respuestas de un sujeto y el sujeto ajuste su comportamiento a dichas circunstancias. Esto puede verse también en los casos de auto-hipnosis donde el individuo funge, simultáneamente, como sujeto y operador. Hull (1933) mencionó el papel de los actos simbólicos en la hipnosis: "actos de puro-estímulo, actos cuya función como estímulo puramente es evocar otros actos" (P.395). Esto implica que se requieren dos momentos distintos para evocar las reacciones en el sujeto: un momento actual donde se evoca un acto y el otro momento, donde ocurrió el acto que es evocado. Hull (1933) señaló que los actos de puro-estímulo constituían lo que en hipnosis era denominado como sugestión, que tiene como consecuencia evocar reacciones específicas en el sujeto.

Al requerirse de dos momentos de respuesta en los contactos de la interrelación, se involucra un actuar como si se respondiera a circunstancias 
o aspectos distintos a los presentes. Los eventos de fSR involucran distintos tipos de actos como si, a saber: a) el individuo puede actuar como si las características de los objetos inmediatos fueran distintas a las características inmediatas, b) responder a objetos ausentes como si estuvieran presentes, c) actuar como si algo ausente estuviera presente; o, d) responder a objetos presentes como si estuvieran ausentes (Ribes \& López, 1985).

Jaynes (1976) señaló acerca del comportamiento hipnótico que "Es un 'como si' que oculta a un 'eso no está'..." (p. 388). En otras palabras, implicaría restringir los ajustes conductuales hacia una relación funcional estímulo-respuesta específica, mientras se omiten todas las posibles relaciones funcionales del mismo tipo que participan simultáneamente. Sarbin y Coe (1972) indicaron que los sujetos que habían reportado alta hipnotizabilidad según las escalas de medición, mostraban un mayor ajuste conductual a situaciones donde se les pedía actuar como si algo diferente a lo inmediato les ocurriera (i.e., competencia para adoptar un rol en términos teatrales). De igual manera, Skinner (1957) expresó que el comportamiento hipnótico involucraba un actuar como si se respondiera a una situación distinta a la inmediata.

3- El desligamiento funcional de propiedades situacionales espacio-tiempo de los eventos con los que se interactúa. En las situaciones hipnóticas, el sujeto actúa como si estuviera respondiendo a una cosa y no otra. El estímulo convencional media las interacciones que no dependen de los aspectos físico-químicos ni biológicos de los elementos inmediatos. De esta manera, mientras en un evento ordinario la presencia o ausencia de un elemento en un determinado arreglo contingencial, que debido a sus propiedades físico-químicas y convencionales (i.e. referentes a propiedades situacionales inmediatas) evocaría un determinado tipo de reacción (como un conjunto de aspectos efectivos y no efectivos o afectivos), en una situación hipnótica ocurriría lo contrario: actuar frente a la presencia de dicho elemento como si no estuviera, o actuar frente a la ausencia de dicho elemento como si se encontrara presente.

4- La emergencia de relaciones de condicionalidad que no dependen de propiedades físicoquímicas y biológicas de los eventos y elementos de respuesta involucrados. Los fenómenos hipnóticos demuestran una sustitución de relaciones de contingencia, no sólo una sustitución de eventos por medio del lenguaje. De ahí que surjan relaciones de condicionalidad que no dependen de las propiedades inmediatas (físico-químicas y biológicas) de los eventos y elementos de respuesta involucrados. Por ejemplo, en el caso de una alucinación, el sujeto hipnotizado no responde sólo en términos efectivos como si el elemento alucinado estuviera presente, mientras las afectaciones pudieran omitirse, tal como lo hace un actor, capaz de interpretar acciones que no le ocurren. El individuo ajusta su comportamiento efectivo hacia la alucinación mientras padece las afectaciones de la interacción como si el objeto alucinado realmente estuviera en frente de él; tal sería el caso de una persona que siendo alérgica a una sustancia, cree que la ha consumido y termina en la sala de urgencias, al borde de una condición letal, hasta que le informan que los análisis químicos no arrojan evidencias sobre el problema que refiere. La articulación de aspectos efectivos y no efectivos del sujeto hipnotizado, permite diferenciar el comportamiento hipnótico de las simulaciones, como se ha demostrado en el caso de los experimentos sobre analgesia hipnótica (Hilgard \& Hilgard, 1975).

En adición a los aspectos que apoyarían la interpretación del comportamiento hipnótico como un evento de fSR, se debe señalar que el comportamiento hipnótico tiene sentido en una situación (i.e., un sistema de contingencias), donde el discurso que utiliza un operador delimita los parámetros y las acciones que un sujeto realizará para conformar ajustes conductuales que pueden excluir otras formas potenciales de relación estímulo- respuesta (e.g., Castellanos, 2009; González-Ordi \& Miguel-Tobal, 1994; Hilgard \& Hilgard, 1975; Hull, 1933; Kantor, 1924; Skinner, 1957). En este tipo de situación se pueden apreciar tres aspectos que diferencian al comportamiento hipnótico de otros tipos de eventos que podrían catalogarse como pertenecientes a la fSR:

1. El comportamiento del individuo se afecta mediante el lenguaje (e.g.; Barber, 1969; Hull, 1933; Kantor, 1924; Pavlov, 1929; Platonov, 1959; Skinner, 1957; Wundt, 1892).

2. En comparación a una situación similar, hay 
una contradicción entre la conducta del individuo y el criterio de ajuste habitual de acuerdo a las relaciones convencionales, es decir, una funcionalidad paradójica (Pavlov, 1929).

3. Se forma un sistema de relaciones estables, o "estado" en términos psicológicos (Ribes, 2007), que se basa en el aspecto no efectivo del comportamiento del individuo.

La consideración de estos tres aspectos lleva a plantear que, cuando se habla de comportamiento hipnótico, se hace referencia a un evento de Afectación Paradójica Estable (o evento APE), en el que se afecta el comportamiento del individuo mediante el lenguaje, mostrándose una contradicción entre la conducta del individuo y el criterio de ajuste habitual (de acuerdo a las relaciones convencionales), al mismo tiempo que se forma un sistema de relaciones estables (o estado, en términos psicológicos) que se basan en el aspecto no efectivo del comportamiento del individuo, excluyéndose formas potenciales de de relación estímulo-respuesta.

El análisis del comportamiento hipnótico o evento APE, como un caso de fSR, supondría la existencia simultánea de dos situaciones que articulan la ocurrencia de los fenómenos hipnóticos: por un lado, la situación convencional que establece las respuestas comunes y/o habituales ante los elementos potencialmente funcionales que se encuentran presentes y, por el otro, la situación operador-sujeto, que incita la ocurrencia de un ajuste conductual que contradice al promovido en la primera situación. De esta forma, las alucinaciones, la anestesia/analgesia, la catalepsia o la amnesia, corresponderían a eventos donde el individuo reacciona como si le sucediera lo opuesto a lo que debería de ocurrirle, mientras los elementos potencialmente funcionales continúan presentes en la situación (i.e., percibir algo que no está o dejar de percibir algo inmediato, no padecer sensaciones o dolor ante determinadas condiciones que usualmente lo provocarían, carecer de movilidad en una parte del cuerpo sin una razón aparente, ser incapaz de recordar algo que acaba de ocurrir). Bajo esta lógica, el comportamiento hipnótico o evento APE, involucra interacciones sustitutivas referenciales (Ribes \& López, 1985; Ribes, 2012): un evento donde las relaciones de contingencia que son transferidas
- desde otra situación - mediante el lenguaje por un mediador, guían los ajustes conductuales del mediado, sustituyendo las contingencias de la situación inmediata.

En este sentido, Torres et al. (2012) señalan que la definición de un sistema contingencial a partir de propiedades convencionales, requiere un contacto de ocurrencia por traslación de la ubicación de las formas de relación pertinentes. Esto implica la articulación de una situación caso, que parte de la existencia de dos sistemas de contingencias que están vigentes de manera simultánea. Para efectos de la presente exposición, se abordará al fenómeno de la Anestesia/Analgesia Hipnótica como una situación caso de fSR, de acuerdo al planteamiento de Torres et al. (2012)-.

\section{La Anestesia/Analgesia Hipnótica - $A / A H$ - como una situación caso de la fSR}

En general, parece haber un acuerdo al señalar que la Anestesia/Analgesia Hipnótica (A/AH) consiste en un evento donde, convencionalmente, se supondría que el individuo debería de manifestar signos de dolor mediante el tipo de estimulación que recibe (i.e., mediante intervenciones, tratamientos o padecimientos), pero debido a la inducción de un estado hipnótico, la persona se mantiene inmutable y sin dolor (e.g., Brown \& Vogel, 1938; Dynes, 1932; Erickson \& Rossi, 2012; Hilgard, 1992; Hilgard \& Hilgard, 1975; Hull, 1933; Kroger, 1963; Patterson \& Jensen, 2003; Platonov, 1959; Sarbin \& Coe, 1972; Stoelb, Molton, Jensen \& Patterson, 2009; Wundt, 1892;).

El análisis de la A/AH, como situación caso, requiere de dos sistemas contingenciales que se presentan simultáneamente: por un lado, la situación que puede ser entendida como "dolor" (Véase la Figura 1), y por el otro, la situación entendida como "hipnosis" (Véase la Figura 2).

En la Figura 1 se puede apreciar la articulación de un sistema contingencial basado en relaciones convencionales, donde existe la función de un Elemento Nocivo que actúa (que está en acto) o puede llegar a actuar (está en potencia). Esta función del Elemento Nocivo promueve una incitación fisiológica de una respuesta conductual en la función de un Sujeto Doliente (Sujeto D). La función del Sujeto D se estructura a partir de una reactividad fisiológica y convencional, así como 


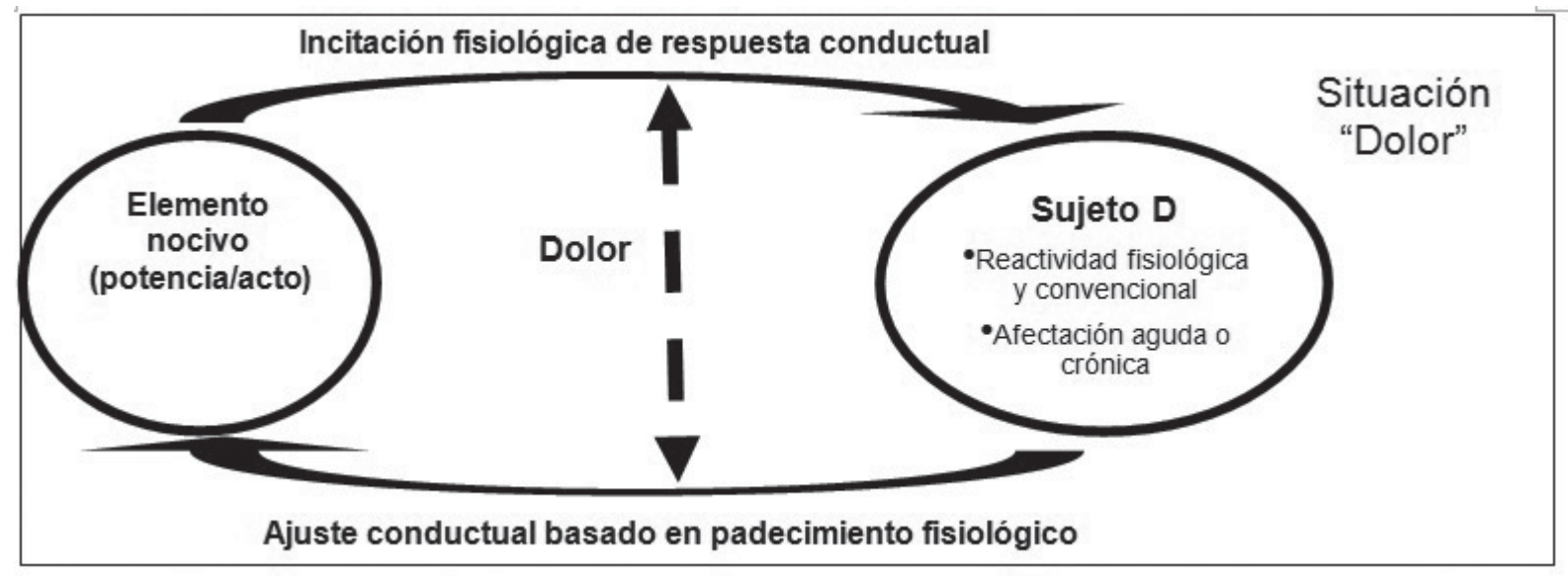

Figura 1. Elementos funcionales que articulan la situación "Dolor".

de la disposición para ser afectado en términos agudos o crónicos. La reactividad y disposición que integran la función de Sujeto D promueve un ajuste conductual (basado en el padecimiento fisiológico) hacia la función del elemento nocivo. La relación que hay entre la incitación fisiológica de la respuesta conductual y el ajuste basado en el padecimiento fisiológico se puede denominar como "dolor".

En la Figura 2 se muestran los elementos funcionales que articulan una situación hipnótica.
La función del operador, estructurada a partir de la competencia lingüística y la aptitud en el manejo de ciertas técnicas, promueve o induce de manera explícita determinadas circunstancias para la función del Sujeto Hipnótico (Sujeto H). La función del Sujeto $\mathrm{H}$, que se estructura a partir de la competencia lingüística y la disposición para mantener una afectación estable, exhibe respuestas pertinentes a las circunstancias promovidas por la función del operador. La relación que hay entre las circunstancias que la función

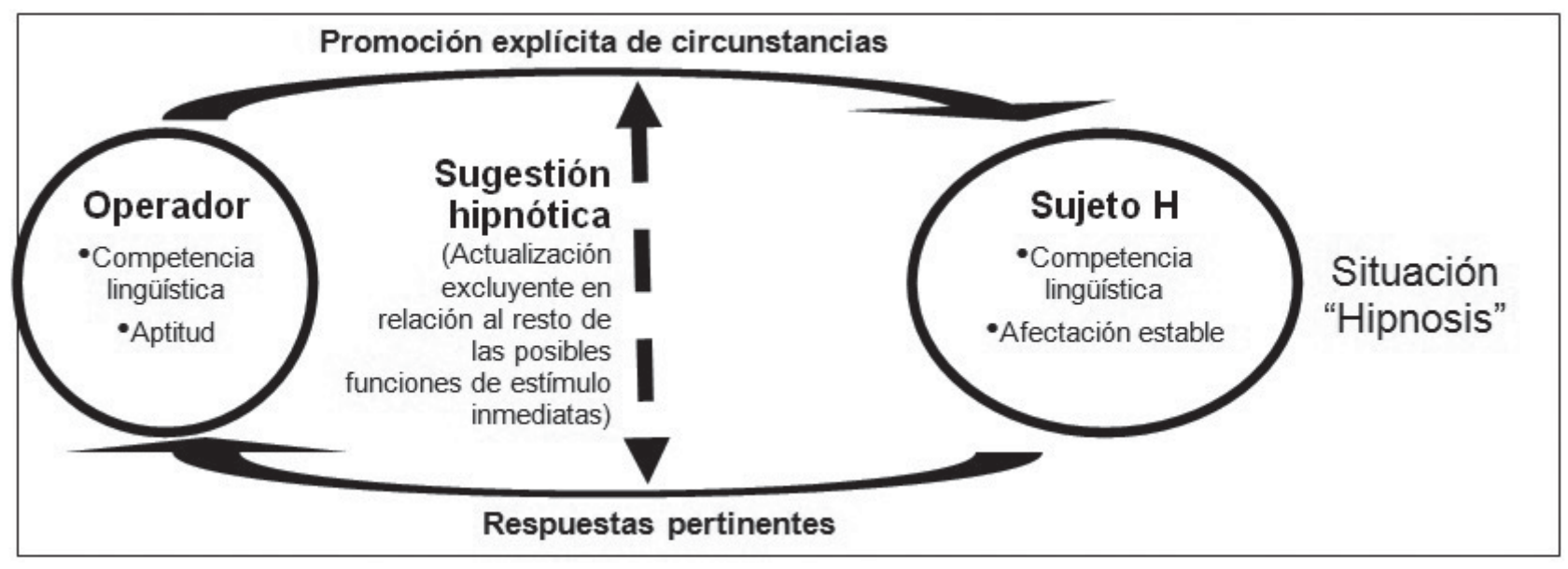

Figura 2. Elementos funcionales que articulan la situación "Hipnosis". 


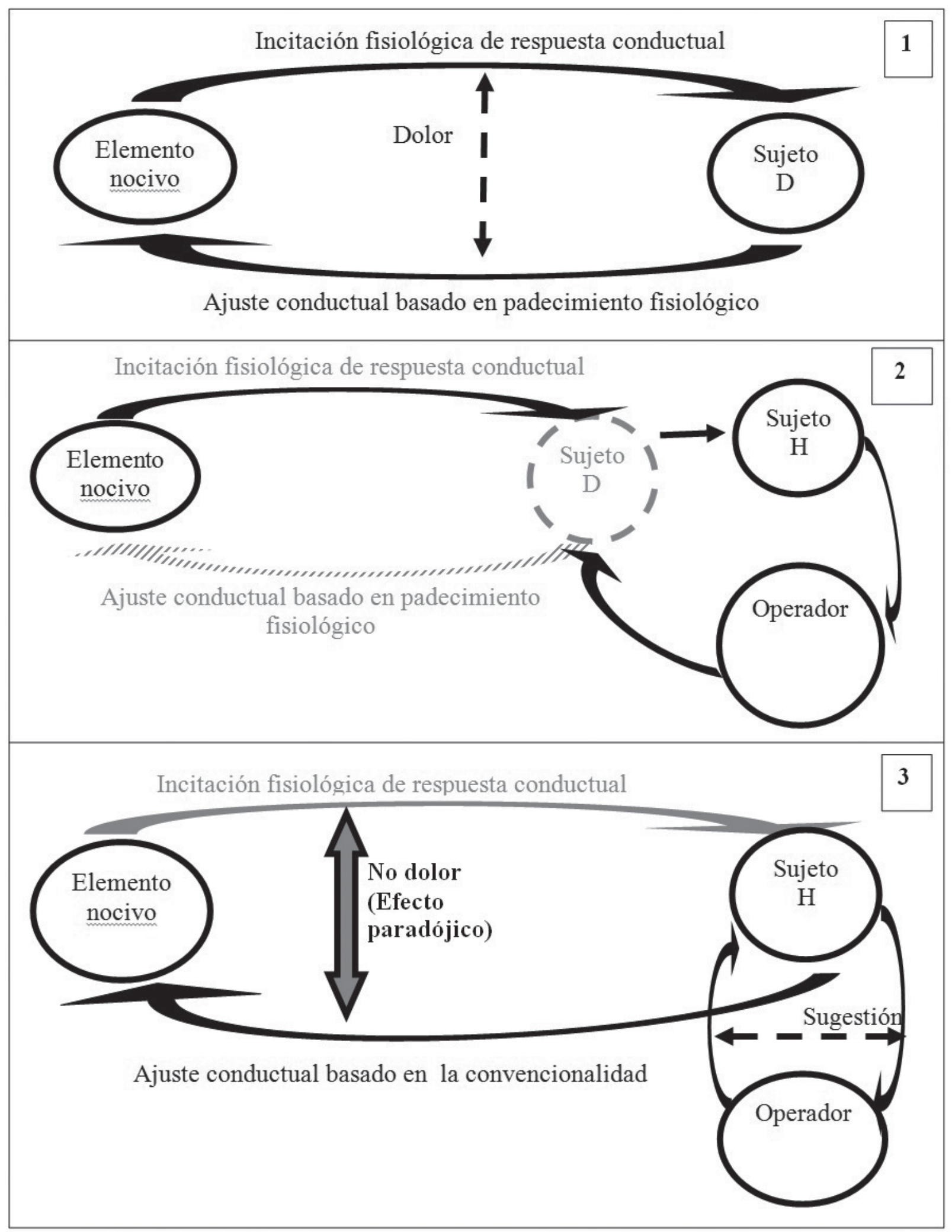

Figura 3. Secuencia de articulaciones de los elementos funcionales que integran a la anestesia/ analgesia hipnótica como situación caso. 


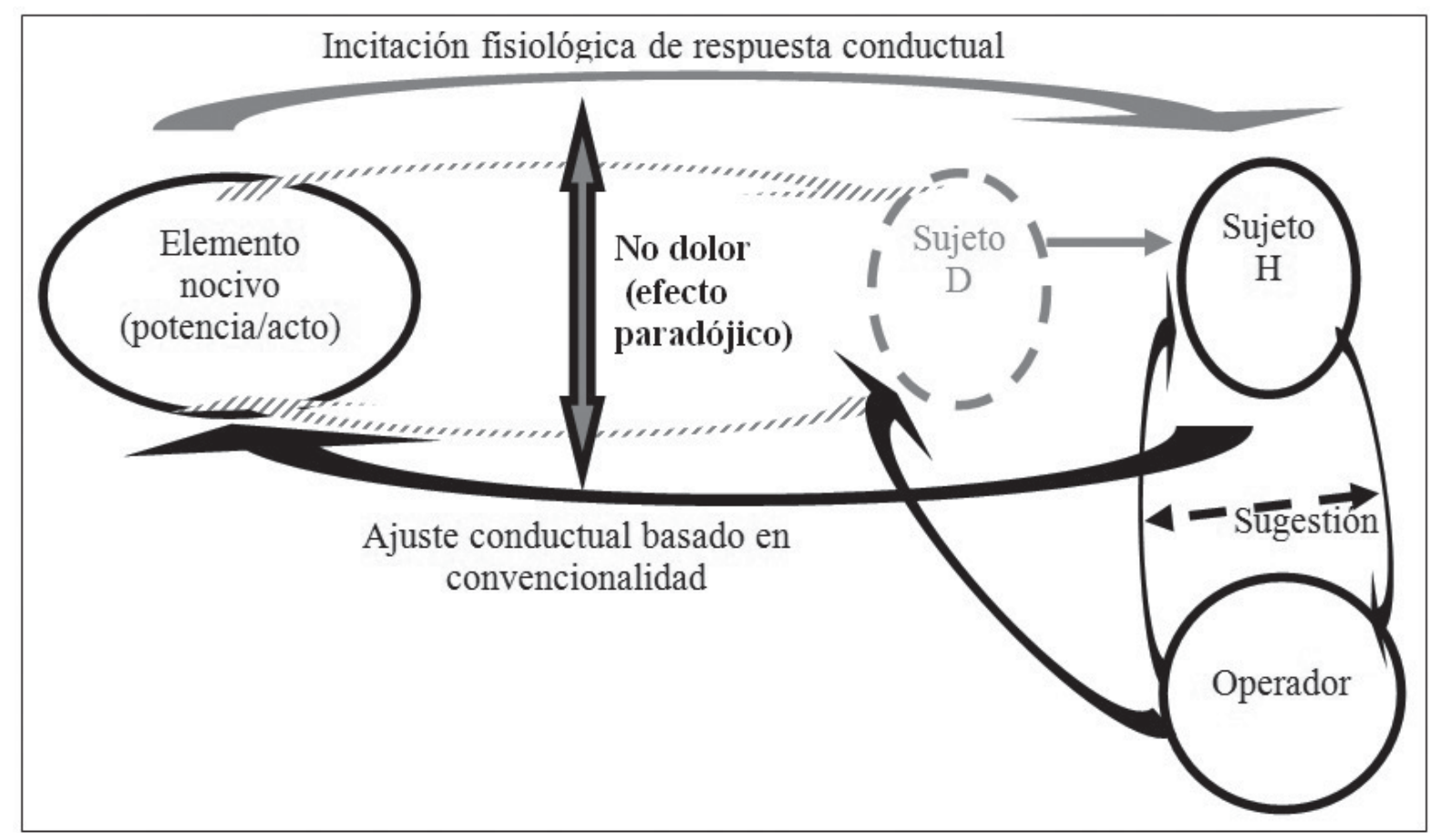

Figura 4. Esquema general de la anestesia/analgesia hipnótica como situación caso.

de operador induce o promueve y las respuestas pertinentes de la función del Sujeto $\mathrm{H}$ se puede Ilamar Sugestión Hipnótica, que implica una actualización de la función de estímulo en torno a los elementos que se presentan en esta misma relación y que excluye al resto de las posibles funciones de estimulo que se encuentran de manera inmediata en la situación.

Cuando la Situación "Dolor" y la Situación “Hipnosis" coexisten en un solo sistema contingencial, se articula el fenómeno de la anestesia-analgesia hipnótica. Este fenómeno se representa en la secuencia de esquemas contenida en la Figura 3.

La Figura 3 muestra la secuencia de articulaciones que dan pie a la anestesia/analgesia hipnótica como una situación caso. En el cuadro 1 se muestra la articulación de la situación "Dolor", como ya se mostró antes (en la Figura 1), mientras que el cuadro 2 presenta la introducción de la función de operador en la situación de dolor (Ver también figura 2). La función de operador promueve las circunstancias para que la función del Sujeto D (Sujeto Doliente) cambie por la función de Sujeto H (Sujeto Hipnótico). La función del elemento nocivo incita fisiológicamente una respuesta con- ductual, pero el ajuste conductual basado en el padecimiento fisiológico no logra completarse porque el lugar de la función del Sujeto $D$ fue tomado por la función del Sujeto H. El cuadro 3 hace explícito que los elementos funcionales de la situación hipnótica (Sujeto $\mathrm{H}$, Operador y Sugestión) promueven un ajuste conductual basado en la convencionalidad que sustituye al ajuste basado en el padecimiento fisiológico. Es por esto que hay un efecto paradójico: mientras la función del elemento nocivo continúa la incitación fisiológica de una respuesta conductual, se presenta un ajuste basado en la convencionalidad que es contrario a lo esperado. La visión general de esta secuencia se muestra en la Figura 4 donde se presenta el panorama general de la articulación de la anestesia/analgesia hipnótica como una situación caso de fSR. La relación Operador-Sujeto $H$, que implica la articulación de la Sugestión, constituye un referente que media la interacción de la función del sujeto D con el Elemento Nocivo.

Aunque la función del elemento nocivo permanece, la función del Sujeto D se sustituye por la función del Sujeto H: se sustituye una contingencia basada en la incitación fisiológica de una 
respuesta conductual que evoca un ajuste conductual basado en el padecimiento fisiológico, por una contingencia donde se conserva la incitación fisiológica de una respuesta conductual y se realiza un ajuste conductual desde un referente que permite la omisión de cualquier función de estímulo potencial que sea distinta al referente mismo. En palabras de Skinner (1957) “El mundo está, por un tiempo, reducido al estímulo verbal que está prácticamente en completo control del sujeto hipnotizado" (p. 160), a lo que habría que añadir: demostrando una contradicción entre lo que sucede y lo que se supone debería de ocurrir.

La relación de "No dolor" que surge entre la función del Elemento Nocivo y la del Sujeto $\mathrm{H}$, implica una actualización de la función de estímulo y respuesta conductual basada en la convencionalidad, que paradójicamente excluye de manera estable a la Función del Elemento Nocivo, como si no ocurriera; es decir, se articula un evento APE.

\section{Conclusiones}

El abordaje del comportamiento hipnótico, desde una perspectiva interconductista, puede ofrecer un panorama claro acerca de cómo conceptuar el fenómeno de la hipnosis. De inicio, esta postura facilita una distinción entre las situaciones hipnóticas y las situaciones que se parecen, pero no lo son (o situaciones "para-hipnóticas"). Al conceptuar el comportamiento hipnótico en términos de un evento APE, se posibilita la identificación de los factores necesarios para articular las relaciones funcionales comprendidas en este tipo de eventos, así como uno de los rasgos distintivos del problema de la hipnosis (la afectación paradójica) que la distingue de la ocurrencia de otros eventos (i.e. relaciones sociales de poder que podrían identificarse de acuerdo al lenguaje ordinario como "sugestión", "influencia", "incitación", por mencionar algunas). Por consiguiente, aquellos sistemas contingenciales que se estructuran mediante la omisión de algún o algunos factores necesarios, por definición no corresponderían a una situación hipnótica. Por ejemplo, cuando se habla de la hipnosis sin necesidad de una inducción formal o explícita (e.g., Erickson et al., 1976; Haley, 1994) o sin necesidad de inducir y evocar un estado hipnótico (e.g., Barber, 1969), se estarían planteando eventos que calificarían en situaciones para-hipnóticas, pues si bien exhiben fenómenos conductuales propios de las situaciones hipnóticas, la forma en que se articula la situación es diferente. Adicionalmente, podrían elaborarse aportaciones innovadoras en el abordaje de eventos relacionados con la forma en que el juego de articulaciones lingüísticas mencionado por un referidor, y no por otro, altera la manera en que un individuo es afectado por las posibles relaciones funcionales de los elementos ambientales o cómo es que el discurso de un individuo puede delimitar la funcionalidad de otros en un ambiente específico. Las implicaciones podrían afectar campos relacionados con el análisis del comportamiento en contextos políticos, artísticos, científicos, filosóficos, entre otros.

Ahora bien, la situación caso (Anestesia/Analgesia Hipnótica), expuesta mediante esta perspectiva interconductual, podría facilitar el entendimiento de por qué el uso del lenguaje puede deshabilitar relaciones funcionales que parecieran "inmutables", si se considera una afectación meramente situacional (por la inmediatez de las propiedades físico-químicas y el respaldo de los aspectos convencionales). Esto representaría una respuesta a un planteamiento hecho por Bernheim (1887): "El hipnotismo no puede ser erigido en método general de anestesia quirúrgica; no puede remplazar al cloroformo, porque las preocupaciones ansiosas que agitan el espíritu de los enfermos en el momento de una operación, impiden con frecuencia la concentración psíquica necesaria al desarrollo de un estado hipnótico" (p. 16). Esto sugiere que la sustitución contingencial que se presenta en los eventos APE, involucra una relación lingüística que no todos los sujetos pueden articular y/o mantener de forma estable.

Al respecto, González-Ordi y Miguel-Tobal (1994) han señalado la discrepancia entre los resultados de los estudios acerca de la hipnosis en el área clínica y el área experimental, debido a los aspectos que intervienen en cada tipo de evento (i.e. no es lo mismo participar en un experimento - donde uno supone que puede o no ocurrir algo-, que solicitar un tratamiento clínico - al que uno le atribuye ciertas propiedades terapéuticas, de acuerdo a un padecimiento específico-). De acuerdo con los elementos que se proponen en 
este escrito, a partir de un marco interconductista (Ribes \& López, 1985; Ribes, 1990b), las diferencias que hay entre los hallazgos del área clínica y la experimental no son el tipo de cosas que se puedan resolver mediante la traducción de los fenómenos clínicos a condiciones experimentales; son cosas que se resuelven mediante la conceptuación del evento psicológico susceptible al control y no a la eliminación de variables.

Para finalizar, es necesario remarcar que el abordaje del comportamiento hipnótico, como un evento APE desde una perspectiva interconductista, involucra el diseño de métodos que sean congruentes para demostrar la lógica de los eventos psicológicos como ocurrencias intra-sujeto (Ribes y López, 1985; Ribes, 2005). Si la afectación, en este caso, paradójica (de acuerdo a circunstancias similares) implica en cada individuo un conjunto de aspectos idiosincrásicos (cada individuo padece el dolor de manera diferente), entonces el análisis conductual debería de concentrarse en ese tipo de aspectos. Aunque las afectaciones sean susceptibles de agruparse según la convencionalidad, no es pertinente el predominio de un trabajo estadístico para valorar las conductas, debido a que se requiere, de inicio, la comprensión de las situaciones que se articulan de acuerdo al comportamiento de cada individuo.

\section{Referencias}

Barber, T.X. (1969). Hypnosis. A scientific approach. USA: Litton Educational Publishing

Bernheim, H. (1887). De la sugestión y de sus aplicaciones a la terapéutica. España: José Plaza y Castaños Editor.

Brown, R., \& Vogel, V. (1938). Psycho-physiological reactions following painful stimuli under hypnotic analgesia contrasted with gas anesthesia and novocain block. Journal of Applied Psychology, 22, 408-420, available via http://dx.doi.org/10.1037/h0053485

Cangas, A. (1998). Análisis del comportamiento hipnótico, Acta Comportamentalia, 6, 61-70.

Castellanos, F. (2009). Análisis conductual de la anestesia hipnótica como un evento psicológico y la propuesta del perfil hipnótico interactivo (PHI). Tesis de maestría, Universidad de Guadalajara, Centro Universitario de Cien- cias Biológicas y Agropecuarias, Guadalajara, México.

Crawford, H., \& Gruzelier, J. (1992). A midstream view of the neuropsychophysiology of hipnosis: recent research and future directions. In E. Fromm \& M.R. Nash (Eds.), Contemporary hypnosis research (pp 227-266). USA: The Guilford Press.

Dixon, M., \& Laurence, J.R. (1992). Two hundred years of hypnotic research: questions resolved? Questions unanswered!. In E. Fromm \& M.R. Nash (Eds.), Contemporary hypnosis research (pp 34-66). USA: The Guilford Press.

Dynes, J. (1932). An experimental study in Hypnotic Anaesthesia. The Journal of Abnormal and Social Psychology, 27(1), 79-88, available via: http://dx.doi:10.1037/h0073199

Elliotson, J. (1843). Mesmerism. In M.M. Tinterow (Ed), Foundations of hypnosis (pp. 194215). USA: Charles C. Thomas Publisher.

Erickson, M.E., \& Rossi, E.L. (2012). Experimentando la hipnosis (abordajes terapéuticos a los estados alterados). USA: The Milton H. Erickson Foundation.

Erickson, M.H., Rossi, E.L., \& Rossi, S. (1976). Hypnotic realities. USA: Irvington publishers.

Gilman, T., \& Marcuse, F. (1949). Animal Hypnosis. Psychological Bulletin, 46(2), 151-165, available via: http://dx.doi: 10.1037/h0060434

Gonzalez-Ordi, H., \& Miguel-Tobal, J.J. (1994). Datos experimentales versus clínicos en la investigación sobre hipnosis. Psicothema, 6, 27-38.

Gruzelier, J. H. (2000), Redefining hypnosis: theory, methods and integration. Contemporary Hypnosis, 17, 51-70, available via: http://dx.doi: 10.1002/ch.193

Haley, J. (1994). Terapia no convencional. Argentina: Amorrortu.

Hilgard, E.R. (1973). The neodissociation interpretation of pain reduction in hypnosis. Psychological Review, 80, 396-411, available via: http://dx.doi: 10.1037/h0020073

Hilgard, E.R. (1992). Dissociation and theories of hypnosis. In E. Fromm, \& M.R. Nash (Eds.), Contemporary hypnosis research (pp. 69-101). USA: The Guilford Press. 
Hilgard, E.R., \& Hilgard, J. (1975). La hipnosis en el alivio del dolor. México: FCE.

Hull, C.L. (1933). Hypnosis and suggestibility. USA: Crown House Publishing

Jaynes, J. (1976). The origin of the consciousness in the break down of the bicameral mind. USA: Houghton Mifflin Company.

Kantor, J.R. (1924). Principles of psychology. USA: Principia Press.

Kirsch, I. \& Lynn, S.J. (1998). Social-Cognitive alternatives to dissociation theories of hypnotic involuntariness. Review of General Psychology, 2, 66-80, available via http:// dx.doi: 10.1037/1089-2680.2.1.66

Kroger, W. (1963). Clinical and experimental hypnosis. USA: J.B. Lippincott Company.

Morgan, W. (2002). Hypnosis in sport and exercise psychology. In J.Van Raalte \& B. Brewer (Eds). Exploring sport and exercise psychology (pp. 151-181). USA: American Psychological Association.

Morgan, W., \& Stegner, A. (2008). Hypnosis in sport: cases, techniques and issues. In $\mathrm{M}$. Nash \& A. Barner (Eds). The Oxford handbook of hypnosis: theory, research, and practice (pp. 681-696). Great Britain: Oxford University Press.

Ortiz, G., Quintana, C., \& Torres, C. (2009). AIDS toward a system for classifying risk and prevention behaviors. Journal of Behavior, Health \& Social Issues, 1(2), 99-110.

Pates, J., Maynar, I., \& Westbury, T.(2001). An Investigation into the Effects of Hipnosis on Basketball Performance. Journal of Applied Sport Psychology, http://www.informaworld.com/smpp/title $\sim \mathrm{db}=$ all $\sim$ conten $\mathrm{t}=\mathrm{t} 713768823 \sim \mathrm{tab}=\mathrm{issues}$ ist $\sim$ branches $=13$ - v1313, 84-102, available via: http://dx.doi. org/10.1080/10413200109339005

Pates, J., Oliver, R., \& Maynar, I.(2001). The Effects of Hypnosis on Flow States and Golf Putting Performance. Journal of Applied Sport Psychology, http://www.informaworld.com/smpp/title $\sim \mathrm{db}=$ all $\sim$ content $=\mathrm{t} 713768823 \sim \mathrm{tab}=\mathrm{issues}$ list $\sim$ branches $=13$ v1313, 341 - 354, available via: http://dx.doi. org/10.1080/104132001753226238

Patterson, D., \& Jensen, M. (2003). Hypnosis and clinical pain. Psychological Bulletin,
129, 495-521, available via: http://dx.doi. org/10.1037/0033-2909.129.4.495

Pavlov, I. (1929 Traducción al español, Ed. 1997). Los reflejos condicionados España: Morata.

Platonov, K. (1959). The word as a physiological and therapeutic factor. Mosow: Foreign languages Publishing House

Ribes, E. (1990a). Problemas conceptuales en el análisis del comportamiento humano. México: Trillas.

Ribes, E. (1990b). Psicología y salud. México: TriIlas.

Ribes, E. (1992). Sobre el tiempo y el espacio psicológicos. Acta Comportamentalia, 0(1), 77-84.

Ribes, E. (1994). Estados y perspectivas de la psicología interconductual. En L. Hayes, E. Ribes, \& F. López (Eds), Psicología interconductual, contribuciones en honor a J.R. Kantor (pp. 9-18). México: Universidad de Guadalajara.

Ribes, E. (2004). Acerca de las funciones psicológicas: un post-scriptum. Acta Comportamentalia, 12, 117-127.

Ribes, E. (2005). ¿Qué es lo que se debe medir en psicología? La cuestión de las diferencias individuales. Acta Comportamentalia, $13,37-52$.

Ribes, E. (2006). Human behavior as language: some thoughts on Wittgenstein. Behavior and Philosophy, 34, 109-121

Ribes, E. (2007). Estado y límites de campo, medios de contacto y análisis molar del comportamiento: reflexiones teóricas. Acta Comportamentalia, 15, 229-259.

Ribes, E. (2012). Las funciones substitutivas de contingencias. En M. A. Padilla \& R. PérezAlmonacid (Eds.). La función sustitutiva referencial, análisis histórico-crítico/avances y perspectivas (pp. 19-34). USA: University Press of the South.

Ribes, E., \& López, F. (1985). Teoría de la conducta. México: Trillas.

Ryle. G. (1949). El concepto de lo mental. España: Paidos.

Sarbin, T., \& Coe, W.C. (1972). Hypnosis, a social psychological analysis of influence communication. USA: Holt, Rinehart and Winston, Inc. 
Skinner, B.F. (1957). Verbal Behavior. USA: Copley Publishing Group.

Spanos, N.P., \& Coe, W.C. (1992). A socialpsychological approach to hypnosis. In E. Fromm \& M.R. Nash (Eds.), Contemporary hypnosis research (pp.102-130). USA: The Guilford Press.

Stoelb, B., Molton, I., Jensen, M., \& Patterson, D. (2009). The efficacy of hypnotic analgesia in adults: a review of the literature. Contemporary Hypnosis, 26, 24-39, available via: http://dx.doi.org/10.1002/ch.370

Torres, C., Ortiz, G., Rangel, N., \& González, V. (2012). Análisis del concepto de "Situación" en la estructuración de las funciones psicológicas. En M.A. Padilla \& R.PérezAlmonacid (Eds.), La función sustitutiva referencial, análisis histórico-crítico/avances y perspectivas (pp. 149-167). USA: University Press of the South.

Vázquez-Mata, I., Vital-Flores, S., Bravo-González, M.A., Jurado-Cárdenas, S. \& Reynoso-Erazo, L. (2009). Retroalimentación biológica y relajación en pacientes con enfermedad renal crónica terminal en tratamiento de hemodiálisis. Journal of Behavior, Health \& Social Issues, 1(2), Pp.35-43.

Weitzenhoffer, A.M., \& Hilgard, E.R. (1962). Stanford Hypnotic Susceptibility Scale, Form "C". Palo Alto (CA): Consulting Psychologists Press.

Wundt, W. (1892). Hipnotismo y sugestión (traducción por Taimrens Drangs). España: Antonio Roch-Editor.

Yapko, M. (1995). Lo esencial de la hipnosis. España: Editorial Paidós.

Auto-referencias de autor: 3

Auto-referencias de revista JBHSI: 2 